\title{
Adenovirus-mediated expression of Fas ligand induces apoptosis of human prostate cancer cells
}

Tammy E. Hedlund', Sandra J. Meech ${ }^{2}$, Sampathkumar Srikanth ${ }^{3}$, Andrew S. Kraft ${ }^{3,4}$, Gary J, Miller ${ }^{4,5}$, Jerome B. Schaack ${ }^{4,6}$ and Richard C. Duke ${ }^{\star 1,3,4}$

${ }^{1}$ Department of Immunology, University of Colorado Health Sciences Center, Denver, Colorado 80262, USA

2 Department of Pediatrics, University of Colorado Health Sciences Center, Denver, Colorado 80262, USA

${ }^{3}$ Division of Medical Oncology, Department of Medicine, University of Colorado Health Sciences Center, Denver, Colorado 80262, USA

${ }^{4}$ University of Colorado Cancer Center, University of Colorado Health Sciences Center, Denver, Colorado 80262, USA

${ }^{5}$ Department of Pathology, University of Colorado Health Sciences Center, Denver, Colorado 80262, USA

${ }^{6}$ Department of Microbiology, University of Colorado Health Sciences Center, Denver, Colorado 80262, USA

* corresponding author: Dr. Richard Duke, Medical Oncology, Box B-171, University of Colorado Health Sciences Center, 4200 East Ninth Ave., Denver Colorado 80262, USA; tel: 303 315-3303; fax: 303 315-8825; email: Richard.Duke@UCHSC.edu

Received 8.5.98; revised 11.9.98; accepted 11.11.98 Edited by J.C. Reed

\begin{abstract}
Several laboratories have reported on the apoptotic potentials of human prostate cancer $(\mathrm{PC})$ cell lines in response to crosslinking of Fas (CD95/APO-1) with agonistic anti-Fas antibodies. We have re-evaluated the apoptotic potentials of seven human PC cell lines using the natural Fas ligand (FasL) in place of agonistic antibody. First, $P C$ cell lines were tested in a standard cytotoxicity assay with a transfected cell line that stably expresses human FasL. Next, we developed an adenoviral expression system employing 293 cells that stably express crmA, a poxvirus inhibitor of apoptosis, to analyze the effects of FasL when expressed internally by the PC cell lines. Our data suggest that the apoptotic potentials of these cell lines were greatly underestimated in previous studies utilizing agonistic anti-Fas antibodies. Lastly, adenoviral-mediated expression of Fas $L$ prevented growth and induced regression of two human PC cell lines in immunodeficient mice. These preliminary in vivo results suggest a potential use for adenovirus encoding FasL as a gene therapy for PC.
\end{abstract}

Keywords: adenovirus; apoptosis; cell death; CD95; Fas ligand; gene therapy; prostate; prostate cancer; recombinant adenovirus

Abbreviations: FasL, Fas ligand; EGFP, humanized, enhanced, red-shifted jellyfish green fluorescent protein; Ad-mFasL, recombinant adenovirus encoding mouse FasL; Ad-hFasL, recombinant adenovirus encoding human FasL; Ad-EGFP, recombinant adenovirus encoding EGFP; PC, prostate cancer; K562-hFasL,
K562 cells stably transfected with DNA encoding human FasL; K562-neo, K562 cells stably transfected with DNA encoding neomycin phosphotransferase; 293-crmA, 293 cells stably transfected with DNA encoding the poxvirus crmA protein

\section{Introduction}

Fas (CD95/APO-1) is a transmembrane glycoprotein that is related to the receptors for tumor necrosis factor and nerve growth factor. ${ }^{1,2}$ Upon being cross-linked with agonistic antiFas antibodies or Fas ligand (FasL), ${ }^{3}$ Fas initiates a complex signal transduction pathway that, in sensitive cell types, ultimately ends in apoptotic cell death. ${ }^{4}$ The Fas/FasL pathway is probably best recognized for its role in the downregulation of expanded clonal $\mathrm{T}$ lymphocyte populations. In this system, Fas is upregulated within a few hours of $T$ cell activation. ${ }^{5,6}$ Several days later, Fas becomes functional and if the cells continue to be stimulated through the antigen receptor, FasL is also upregulated and the majority of activated cells undergo apoptosis, allowing the immune system to return to its normal resting size and repertoire. ${ }^{4}$

In addition to regulating immune responses, Fas and its ligand are likely to play a role in other systems as well. For example, the testes and placenta, both of which are known to be immune-privileged tissues, express FasL. ${ }^{7,8}$ Additionally, Fas and FasL have been found to be coexpressed in a few epithelial tissues that are marked by apoptotic cell turnover, such as the uterus and prostate. ${ }^{8-10}$ Both of these tissues are steroid-dependent and undergo apoptosis within 24-48 h after hormone depletion. ${ }^{11,12}$ Interestingly, the apoptosis that occurs in response to steroid depletion has recently been shown to require sufficient Fas expression. ${ }^{13,14}$ These data support a role for the Fas signaling pathway in the normal renewal of the uterine and prostatic epithelium.

In addition to the in vivo data above, several laboratories have recently demonstrated a potential role for Fasdependent apoptosis in human prostate cancer (PC) cell lines. ${ }^{15-17}$ Although Fas expression has proved to be a common feature of the cell lines studied, contradictory results were reported with regards to their apoptotic potentials. ${ }^{15-17}$ This may be due to the use of different agonistic anti-Fas antibodies among laboratories, or to the different experimental conditions that were employed.

Preliminary data in our laboratory suggested that the apoptotic potential of at least one PC cell line was markedly increased when the cells were coincubated with a transfected leukemia cell line that stably expresses human FasL. For this reason, we began re-evaluating the sensitivities of the seven PC cell lines used previously ${ }^{17}$ to determine if the use of agonistic IgM anti-Fas antibodies commonly under-estimated or mis-estimated the apoptotic potential of these cells. We next developed an adenoviral 
method of gene transduction to study the effects of FasL when expressed internally by the PC cells. This system was optimized by the use of 293 cells that were stably transfected with DNA encoding the poxvirus crmA protein ${ }^{18}$ to provide them with resistance to FasL-mediated apoptosis, thus increasing the efficiency of virus production. Lastly, we present preliminary in vivo evidence for a potential gene therapy approach for PC using recombinant adenovirus encoding FasL.

\section{Results and Discussion}

\section{PC cell lines show enhanced sensitivity to hFasL versus agonistic antibody}

Prostate cancer cells were labeled with ${ }^{51} \mathrm{Cr}$ and were coincubated for $18 \mathrm{~h}$ at various effector-to-target ratios with either K562-hFasL or K562-neo. Each of the seven PC targets was tested simultaneously in cytotoxicity assays to allow for direct comparison of their apoptotic potentials. L1210-Fas cells served as positive controls since they are highly sensitive to Fas crosslinking. ${ }^{24}$ As shown in Figure $1 \mathrm{~A}$, K562-hFasL induced lysis of L1210-Fas in a dose dependent fashion whereas no specific lysis was detected when K562 cells transfected with neomycin phosphotransferase alone were used as effectors. When used as a target, the PC cell line ALVA-31 behaved similarly (Figure 1B), with cell lysis reaching $63 \%$ at an effector to target $(E: T)$ ratio of $10: 1$. A summary of the results with all eight target cell lines is presented in Table 1. The cell lines are listed in order of descending apoptotic potentials as observed in the cytotoxicity assay. Also presented in Table 1, are the results from previous studies using an agonistic mouse IgM anti-hFas antibody. ${ }^{17}$ These results demonstrate a markedly enhanced activity of membrane-expressed hFasL versus agonistic antiFas antibody.

An interesting feature of the prostate is that it is believed to escape immune surveillance to some extent 'because it lacks afferent lymphatics and because of the immunosuppressive properties of seminal fluid' ${ }^{31}$ In fact, the normal prostatic epithelium has been found to co-express Fas and FasL, as indicated by studies with both mouse $\mathrm{e}^{9,10}$ and human ${ }^{8}$ prostatic tissue. Therefore, it may be more relevant to test the sensitivities of the PC cell lines to internally expressed FasL through transgene methods of gene expression, rather than to FasL-expressing effector cells. Given that FasL expression was expected to induce apoptosis, we chose to develop an adenoviral system of gene transduction rather than to establish stably transfected cell lines or to rely on the limited efficiency of transient transfection methods. Adenovirus entry has been shown to be highly efficient in cells that express the integrin family of adhesion molecules ${ }^{32,33}$ and this is a common feature of many PC cell lines. ${ }^{34,35}$

\section{Construction of recombinant adenovirus encoding FasL and propagation in 293-crmA cells}

cDNA encoding $\mathrm{mFasL}$ was inserted into the $\mathrm{E} 1$ region of a replication deficient human adenovirus 5 construct under the control of the CMV immediate early promoter. Initial attempts

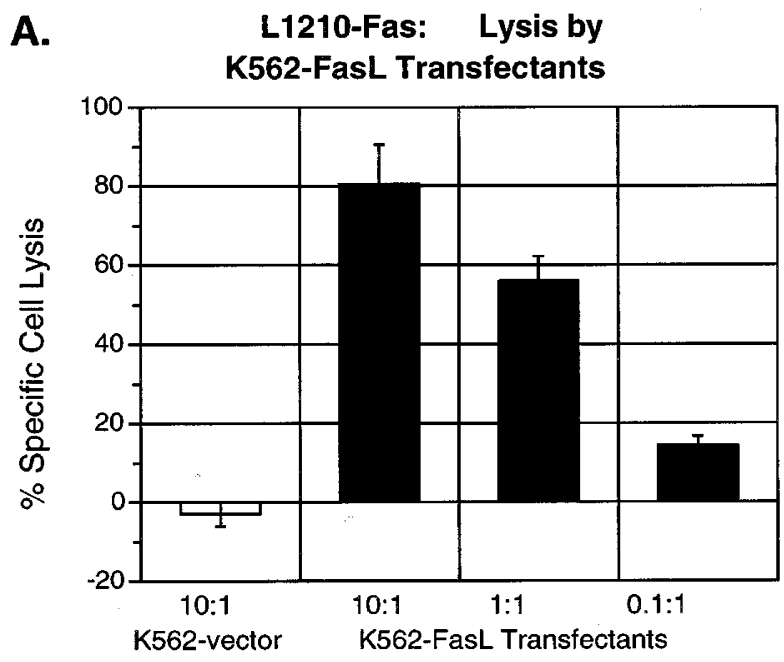

B.

ALVA-31: Lysis by K562-FasL Transfectants

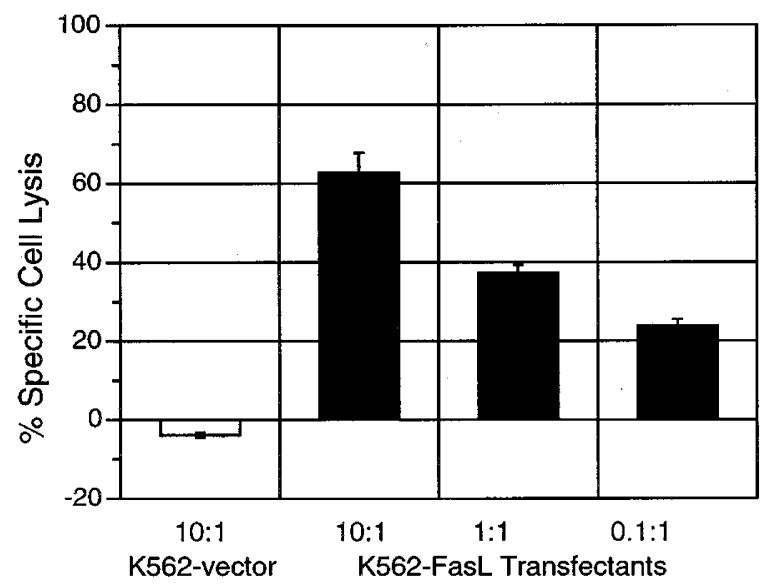

Figure 1 The dose-dependent lysis of (A) L1210-Fas or (B) ALVA-31 target cells by K562 human leukemia cells stably transfected with hFasL. Lysis was measured using a ${ }^{51} \mathrm{Cr}$-release assay after an $18 \mathrm{~h}$ coincubation of target and effector cells. Each value represents the mean of triplicate samples \pm S.D. Spontaneous ${ }^{51} \mathrm{Cr}$ release was $22 \%$ for L1210-Fas and $27 \%$ for ALVA-31

Table 1 Specific lysis of human prostate cancer cell lines by K562-FasL transfectants at a 10:1 effector to target ratio. A comparison with cell lysis achieved using an anti-Fas IgM antibody in previous studies*

\begin{tabular}{lcc}
\hline $\begin{array}{l}\text { Target } \\
\text { cell line }\end{array}$ & $\begin{array}{c}\text { \% Lysis by } \\
\text { K562-Fasl }( \pm \text { S.D.) }\end{array}$ & $\begin{array}{c}\text { \% Lysis by anti-Fas } \\
\text { lgM antibody }\end{array}$ \\
\hline L1210-Fas & $80 \pm 10$ & (not determined) \\
ALVA-31 & $63 \pm 5$ & $28 \%$ \\
TSU-Pr1 & $52 \pm 13$ & undetectable \\
PPC-1 & $48 \pm 1$ & $10 \%$ \\
JCA-1 & $47 \pm 6$ & $10 \%$ \\
LNCaP & $27 \pm 3$ & undetectable \\
DU 145 & $21 \pm 2$ & undetectable \\
PC-3 & $19 \pm 5$ & undetectable \\
\hline
\end{tabular}

${ }^{*}$ Previously published data. ${ }^{17} \sharp \mathrm{JCA}-1$ and DU 145 showed slight but statistically significant lysis by the vector-transfected K562 clone $(14 \pm 1$, and $4 \pm 0.2 \%$, respectively) 
to propagate the virus in the 293 cell line resulted in early death of the cells before significant viral titers could be obtained (data not shown). Greatly reduced viral titers were also reported by Muruve et al. ${ }^{36}$ in a similar attempt. We suspected that 293 cells may be undergoing apoptosis before significant viral replication occurred. To test this hypothesis, 293 cells were analyzed for Fas expression by flow cytometric immunofluorescence (Figure 2A). The mean Fas fluorescence was 4.9-fold greater than nonspecific fluorescence. This value is relatively high compared to other cell lines that we have tested using the same method, and is similar to the level of Fas expressed by CEM cells. ${ }^{17}$ Subsequent cytotoxicity experiments indicated that 293 cells are extraordinarily sensitive to the lytic effects of K562-

A.

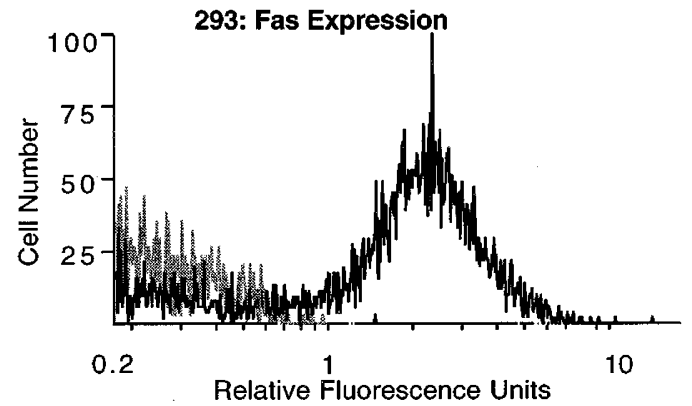

B.

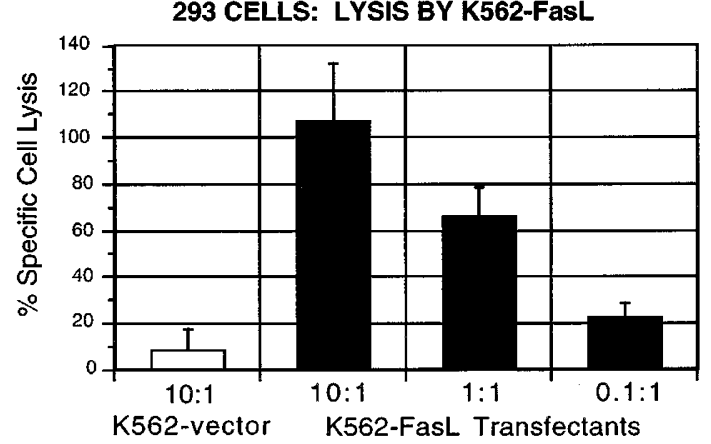

C.

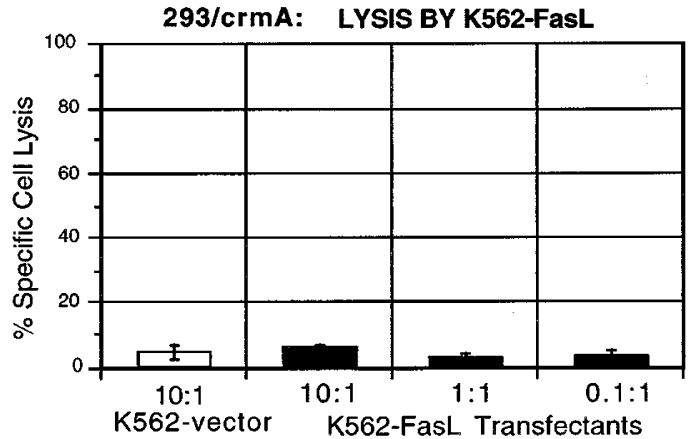

Figure 2 (A) Flow-cytometric analysis of Fas expression by 293 cells. Surface Fas expression was detected using an FITC-conjugated mouse IgG1 anti-human Fas antibody (black peak). Nonspecific fluorescence was estimated using an isotype-matched control antibody directed against a non-mammalian protein (gray peak). (B) Specific lysis of 293 cells or (C) 293crmA transfectants by K562-hFasL as measured by the ${ }^{51}$-Cr-release assay. Each value represents the mean of triplicate determinations \pm S.D. Spontaneous ${ }^{51} \mathrm{Cr}$ release was $41 \%$ for 293 cells and $32 \%$ for 293 -crmA transfectants
hFasL (Figure 2B), with complete lysis occurring at an E:T ratio of $10: 1$. Together, these data support the hypothesis that 293 cells were undergoing FasL-mediated apoptosis during our attempts to produce Ad-mFasL virus. To overcome this problem, 293 cells were stably transfected with a plasmid encoding the cowpox virus caspase inhibitor crmA which inhibits FasL and TNF-mediated apoptosis. ${ }^{18}$ The resulting 293-crmA transfectants were almost entirely resistant to K562-hFasL (Figure 2C) allowing us to effectively propagate adenoviruses encoding mouse and human FasL.

\section{Efficiency of adenovirus gene transduction in PC cells}

To be sure that adenovirus-mediated gene expression could be achieved efficiently in the PC cells, each cell line was transduced with 100 p.f.u./cell Ad-EGFP for $1 \mathrm{~h}$. After $24 \mathrm{~h}$ of further incubation, the cells were analyzed for fluorescence by flow cytometry (Table 2). The transduction efficiency was high in six of the seven cell lines, with greater than $90 \%$ of the cells expressing EGFP. LNCaP was the only cell line that showed relatively poor adenoviral gene transduction, as measured by both the per cent positive cells $(61 \%)$, and the relatively low fluorescence intensity obtained (13-fold brighter than control cells). Similar transduction efficiencies were obtained with each of the cell lines using a multiplicity of infection of 10 p.f.u./cell (data not shown). These data indicated that adenoviral transduction would be feasible in at least six of the seven PC cell lines.

\section{Effects of Ad-mFasL transduction on PC cell growth}

To determine how the PC cell lines would respond to internally expressed FasL, the growth of cell monolayers was measured for 1 week after transduction with either Ad-mFasL or AdEGFP as a control. As shown in Figure 3, ALVA-31 cells that were treated with Ad-EGFP show logarithmic growth as they approach confluency. A phase contrast photomicrograph was taken at day 4 post-infection and is shown to the right of the growth curve in Figure 3. Ad-EGFP treatment did not increase apoptosis above basal levels as confirmed by the lack of nuclear fragmentation apparent after staining cells with propidium iodide and Hoechst 33342 and observing under a fluorescent microscope (data not shown). In contrast, ALVA-

Table 2 Efficiency of Ad-EGFP expression in seven PC cell lines. EGFP fluorescence was measured both by the per cent positive cells and the fold increase in mean fluorescent intensity as compared to untreated cells

\begin{tabular}{lcc}
\hline Cell line & \%old increase in \\
\hline ALVA-31 & 99.6 & $\begin{array}{c}\text { Foltive cells } \\
\text { mean fluorescence }\end{array}$ \\
JCA-1 & 98.7 & 118 \\
PPC-1 & 98.1 & 172 \\
DU 145 & 97.2 & 235 \\
TSU-Pr1 & 93.2 & 813 \\
PC-3 & 92.2 & 55 \\
LNCaP & 61.2 & 129 \\
\hline
\end{tabular}




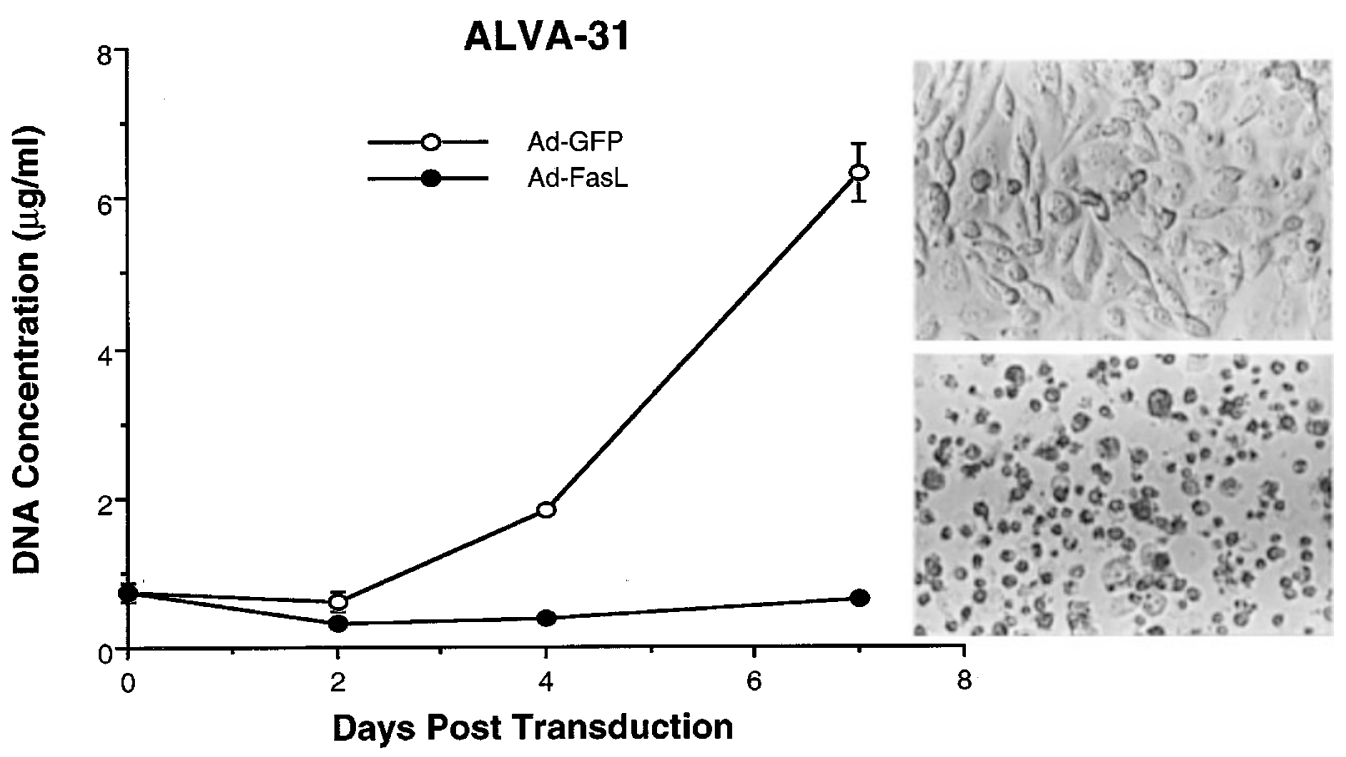

Figure 3 Growth of ALVA-31 cells after adenoviral gene transduction of mFasL or EGFP. Cell monolayers were harvested at the designated time points for DNA quantitation to measure changes in growth. Each value represents the mean of triplicate samples \pm S.E. To the right of each condition is a phase-contrast image of ALVA-31 cells as they appeared at Day 4 of the growth assay

31 cells that were treated with Ad-mFasL were nearly completely apoptotic within 24-48 h (Figure 3) and the few remaining cells failed to resume exponential growth over the course of this assay. Similar results were obtained with recombinant adenovirus encoding human FasL (data not shown). These data support the cytotoxicity results, suggesting that ALVA-31 cells are far more sensitive to the natural FasL protein than they are to agonistic anti-Fas antibody. Furthermore, this cell line appears to be more sensitive to internally expressed FasL than to that presented by K562hFasL.

The short-term growth curves of other PC cell lines after adenoviral transduction are shown in Figure 4. The cell line PPC-1 behaves similarly to ALVA-31 in that FasL transduction nearly obliterates the entire population of cells, and positive growth is not detected over the course of the assay. FasL transduction was to varying extents less effective on JCA-1, PC-3 and TSU-Pr1 cell growth in this in vitro assay. Interestingly, TSU-Pr1 is far more sensitive to FasL when it is presented by the K562-hFasL transfectant than when it is internally expressed. The reasons for this difference are not clear. However, two explanations are possible. First, TSU-Pr1, like certain other prostate cancers, ${ }^{37-39}$ may produce high amounts of matrix metalloproteinases resulting in the production of soluble FasL which is not functional. ${ }^{40-42}$ Second, TSUPr1 may not efficiently trimerize FasL into its functional form. ${ }^{43}$ Investigation of these possibilities will be the focus of future studies. The PC cell lines DU 145 and LNCaP yielded inconsistent results in the short-term growth assays with repeated trials (data not shown). For these reasons, we are currently unable to draw any conclusions regarding the sensitivities of these two cell lines to internally expressed FasL; however, recent results reported by Liu and colleages ${ }^{44}$ suggest that these cell lines constitutively secrete soluble FasL which could interfere with FasL-mediated apoptosis following transduction.

\section{Preliminary studies on the effects of Ad-FasL on prostate tumor growth in vivo}

Although the ALVA-31 cell line appeared to be quite sensitive to apoptotic induction by Ad-mFasL, it was not clear if the few remaining cells would actually be capable of regenerating over a longer period of time, and if the cells would behave similarly in an in vivo environment after FasL transduction. To address these issues, a preliminary experiment was initiated to compare the growth of ALVA31 cells with and without Ad-mFasL after 6 weeks of growth intradermally in $\mathrm{Nu} / \mathrm{Nu}$ mice. Untreated ALVA-31 cells produced tumors in nine of 12 injection sites. However, ALVA-31 cells that were infected with Ad-mFasL (10 p.f.u./ cell) prior to injection failed to produce tumors in any of four sites. Also of interest in this preliminary set of experiments was the observation that the established control tumors could not be eradicated entirely by later injection of Ad$\mathrm{mFasL}$ virus ( $10^{7}$ p.f.u./tumor). Although localized apoptosis was apparent in tissue sections near the injection sites (data not shown), the virus may not have been sufficiently dispersed within the tumor to cause significant regression. This preliminary experiment raised several other important questions. For example, was the lack of tumor growth due specifically to FasL expression, or would the same effect be observed with Ad-EGFP? Also, what are the in vivo effects of Ad-FasL in a prostate tumor cell line that appeared resistant in vitro? To address these issues, a second set of experiments was carried out in mice using the PC cell line TSU-Pr1, whose growth was not inhibited by either Ad-EGFP or Ad-FasL in vitro. 


\section{GROWTH OF PROSTATE CANCER CELL LINES AFTER ADENOVIRAL TRANSDUCTION WITH EGFP OR FAS LIGAND}
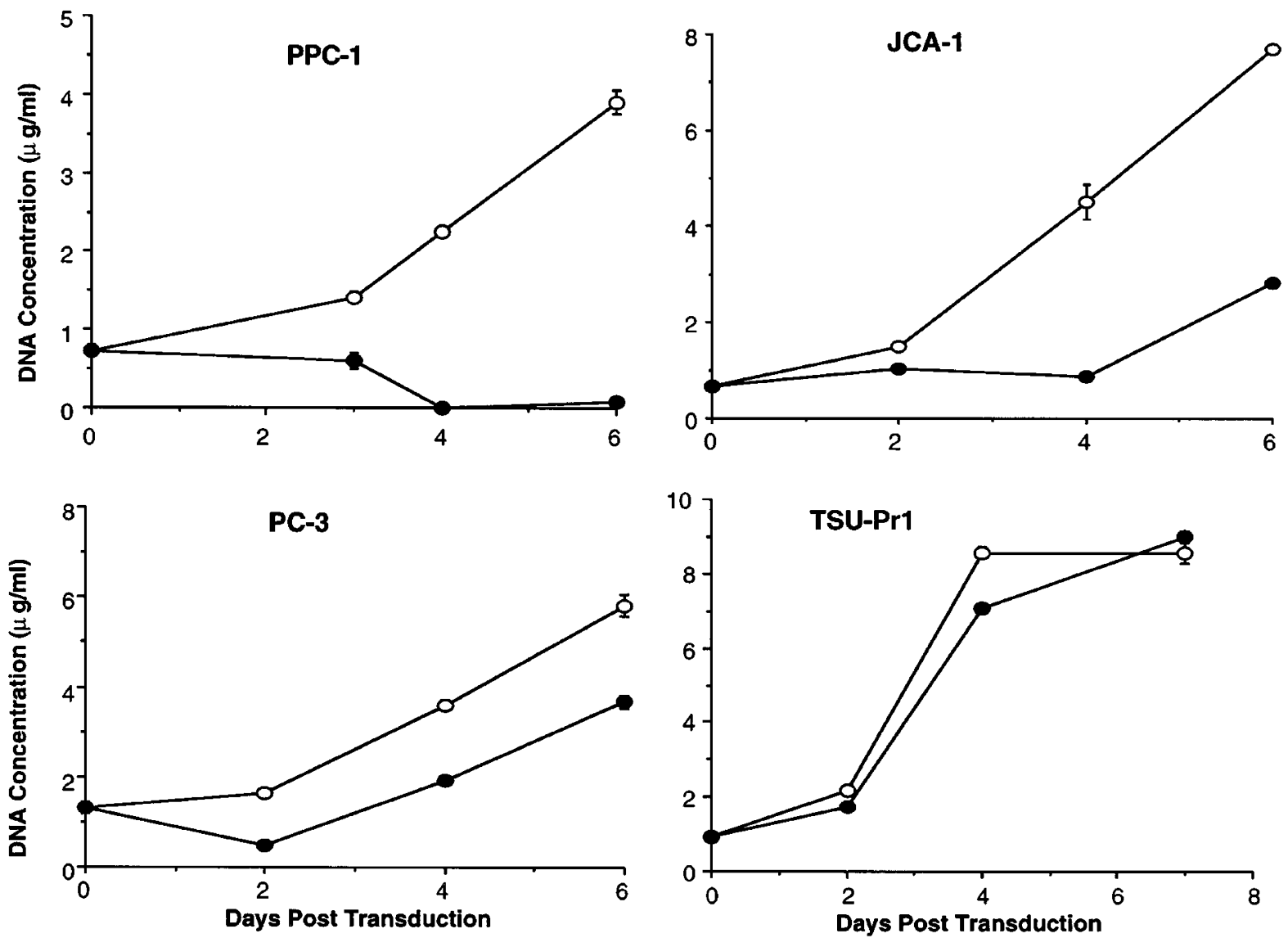

Figure 4 Growth of four prostate cancer cell lines after adenoviral transduction of EGFP or mFasL. The experimental design follows that described for Figure 3

Table 3 The effects of Ad-FasL on growth of TSU-Pr1 cells in nude mice. Cells were untreated (control), or pre-infected with Ad-EGFP or Ad-FasL. For comparison, several established control tumors were injected at day 10 with free Ad-EGFP or Ad-FasL virus

\begin{tabular}{lcc}
\hline $\begin{array}{l}\text { TSU-Pr1 } \\
\text { pretreatment }\end{array}$ & $\begin{array}{c}\text { Day 9 } \\
\text { Mean tumor } \\
\text { area } \pm \text { S.E. }\end{array}$ & $\begin{array}{c}\text { Day 18 } \\
\text { Mean tumor } \\
\text { area } \pm \text { S.E. }\end{array}$ \\
\hline Control & $9.7 \pm 1.9$ & $21 \pm 10.3$ \\
Ad-EGFP & $12.6 \pm 2.3$ & $27.5 \pm 4.3$ \\
Ad-mFasL & $2.3 \pm 0.9$ & $2.3 \pm 0.9$ \\
Injected Ad-EGFP & - & $31 \pm 3.9$ \\
Injected Ad-mFasL & - & $23 \pm 9.2$ \\
\hline
\end{tabular}

TSU-Pr1 cells were pre-infected with Ad-EGFP or AdmFasL as described above for the ALVA-31 cell line, and cells were injected intradermally into $\mathrm{Nu} / \mathrm{Nu}$ mice. After 9 days, when control tumors were established, four control tumors were injected with Ad-EGFP or Ad-mFasL virus. One animal was then sacrificed $24 \mathrm{~h}$ later for histologic analysis. Calipers were used to measure the length and width of each tumor at days 9 and 18. The remaining animals were sacrificed after a total of 18 days. As shown in Table 3, there was no significant difference between the mean sizes of control and Ad-EGFP-infected TSU-Pr1 tumors either at day 9 or 18 . In contrast, Ad-FasL tumors were significantly smaller and no change in tumor size was detected between days 9 and 18. Histologic analyses of these tumors revealed several surprising findings. First, AdFasL-treated tumors, although small, appeared viable ruling out the possibility that scar tissue had completely replaced the tumor cells. Second, both Ad-FasL- and Ad-EGFPtreated tumors had extensive neutrophil infiltration (data not shown). This suggests that the initial infiltration of neutrophils is induced nonspecifically by adenoviral infection of tumor or other cells in the dermis. Furthermore, this infiltration is not in itself responsible for the regression/rejection of tumors in Ad-FasL-treated mice, as the tumors injected with Ad-EGFP were as large and as viable as control tumors. It was observed, however, that the 
neutrophils in the Ad-FasL-treated tumors often appeared apoptotic (data not shown). Thus, it is plausible that once the neutrophils are recruited in response to adenoviral infection, they undergo apoptosis in response to FasL, produced perhaps by dermal cells, and may potentiate a greater inflammatory response that indirectly suppresses TSU-Pr1 growth. Further experimentation will be necessary to better define the role of neutrophils in mediating the rejection/regression of tumor cells that are not intrinsically sensitive to Ad-FasL mediated apoptosis.

\section{Perspective}

During the course of the current study, Arai and colleagues ${ }^{45}$ addressed similar questions regarding the effects of FasL expression on the growth of colon cancer cell lines in mice. Overall, their findings strongly support the therapeutic potential of FasL transgene expression in causing the regression of tumors. Furthermore, their data suggest that this favorable response is not only observed in cell lines that undergo apoptosis in response to FasL expression in vitro. In fact, tumor regression also occurs in a Fas-negative (and FasL-insensitive) cell line, regression is associated with a marked infiltration by neutrophils, many of which appear apoptotic. These results are at least in partial agreement with our preliminary findings in prostate cancer cells. Whether or not this effect is directly due to the actions of neutrophils, it follows that this type of gene therapy might be most useful for the treatment of slow-growing cancers, such as those of prostatic origin. It has been estimated that the doubling times of late stage prostate cancers is typically near 2 years. ${ }^{46}$ Therefore, a therapeutic strategy that can even modestly extend this doubling time, could extend a patient's life by a number of years, especially if initiated soon after diagnosis.

The data presented in the current study indicate that several human PC cell lines are significantly more sensitive to FasL-mediated apoptosis than was originally reported with the use of agonistic anti-Fas antibodies. Furthermore, the majority of these cell lines respond best to FasL when it is expressed internally via the adenoviral system. This method of internal expression may better represent what occurs in the prostate in vivo since the prostatic epithelium has been shown to coexpress Fas and FasL. ${ }^{8-10}$ Our preliminary in vivo experiments, in conjunction with the recent findings of Arai et al. ${ }^{45}$ suggest therapeutic potential for FasL transgene expression in treating cancer patients. Given the prevalence of prostate cancer and the limited effectiveness of available therapies, further research in this area seems warranted

\section{Materials and Methods}

\section{Nontransfected cell cultures}

The human PC cell lines, LNCaP, ALVA-31, TSU-Pr1, JCA-1, PPC1, PC-3 and DU 145 were obtained and maintained as described previously. ${ }^{17} \mathrm{~K} 562$, a human erythroid leukemia cell line ${ }^{20}$ and 293 , a human embryonic kidney cell line transformed by the E1 region of the adenovirus 5 chromosome ${ }^{21}$ were purchased from the American
Type Culture Collection (Rockville, MD, USA) and were maintained in RPMI 1640 (Gibco, Grand Island, NY, USA) supplemented with $7.5 \%$ heat-inactivated FBS (Hyclone, Logan UT, USA) and $2 \mathrm{mM} \mathrm{L-}$ glutamine (Gibco) at $37^{\circ} \mathrm{C}$ in $95 \%$ air and $5 \% \mathrm{CO}_{2}$.

\section{Stably transfected cell lines}

(1) K562-hFasL and K562-neo: human K562 chronic myelogenous leukemia cells were transfected with plasmids encoding human FasL and neomycin phosphotransferase (K562-hFasL) or with a plasmid encoding neomycin phosphotransferase alone (K562-neo) as described elsewhere. $^{22}$ (2) 293-crmA: a modified calcium phosphate precipitation technique ${ }^{23}$ was used to transfect 293 cells with a pcDNA3-crmA construct ${ }^{18}$ generously provided by Dr. V. Dixit (Genentech Inc., South San Francisco, CA, USA). Stable transfectants, resistant to agonistic anti-Fas antibody-mediated apoptosis, were selected with Geneticin (Gibco) and pooled. (3) L1210-Fas: ${ }^{24}$ this transfected mouse lymphocytic leukemia cell line expresses high levels of mouse Fas and was kindly provided by Dr. P. Golstein (Marseilles, France). All transfected cell lines were routinely cultured in the presence of $600 \mu \mathrm{g} / \mathrm{ml}$ active Geneticin, using the medium and conditions described for nontransfected cells.

\section{Adenovirus constructs and viral amplification}

Ad5d/327CMV-EGFP (Ad-EGFP) encodes a humanized, enhanced, red-shifted jellyfish green fluorescent protein $^{25}$ (Clontech Laboratories, Palo Alto, CA, USA) under the control of the CMV major immediate early promoter (J. Schaack et al., submitted). Ad5dl327CMV-mFasL (Ad-mFasL) was generated as follows. In brief, mouse FasL CDNA was generated by $R T-P C R^{7}$ and inserted into the PACCMV plasmid encoding the left end of the Ad5 chromosome but in which the CMV immediate early promoter replaces the E1 region. ${ }^{26}$ Recombinant virus was generated as described previously ${ }^{27}$ by transfecting 293-crmA cells with a mixture of pACCMV-mFasL and Ad5d/327 $7_{\mathrm{Bst}} \beta$-gal DNA-Terminal protein complex prepared from purified virions and digested with Bst-B1 which cleaves uniquely $3^{\prime}$ of the LacZ coding sequence. The transfected cells were incubated until the development of a strong cytopathic effect and freeze-thawed to release virus. Dilutions of the cell lysate were used to infect 293-crmA cells, which were then overlaid with medium containing Noble agar (Difco, Detroit, MI, USA). After plaques developed, the cells were stained with neutral red (Baxter) and X-gal (Boehringer Mannheim, Indianapolis, IN, USA). Plaques that were clear in the presence of X-gal, and thus likely to be recombinants, were picked and grown in 293-crmA cells. Lysates of the plaque-infected cells were used to infect 293-crmA cells. Viral DNAs were isolated ${ }^{28}$ and restriction analysis used to select recombinant viruses encoding $\mathrm{mFasL}$. Recombinant adenovirus encoding human FasL (Ad-hFasL) was generated using a similar strategy.

\section{Adenovirus transduction of PC cells and growth assays}

Cell monolayers were trypsinized and washed once with standard growth medium. For each cell line, $3.2 \times 10^{5}$ cells (control) or $2.4 \times 10^{5}$ cells (Ad-EGFP or Ad-mFasL transduced) were placed into each of three conical Eppendorf tubes. Cells were pelleted by centrifugation, and supernatants were removed by aspiration. The pellets were resuspended in $500 \mu \mathrm{l}$ of either plain medium (negative control), or $500 \mu \mathrm{l}$ of medium containing approximately 10 or 100 p.f.u./cell of Ad$\mathrm{mFasL}$ or Ad-EGFP. Cells were incubated for $1 \mathrm{~h}$ in a $37^{\circ} \mathrm{C}$ water bath 
with periodic mixing and were then washed twice with medium. The final cell pellets were resuspended in $16 \mathrm{ml}$ medium (control cells) or $12 \mathrm{ml}$ medium (Ad-mFasL and Ad-EGFP transduced cells). Each sample was then aliquotted into quadruplicate wells (1 $\mathrm{ml}$ each) of three 24-well tissue culture plates. To quantify the amount of DNA in the initial number of cells plated (i.e. Day 0 ), $1 \mathrm{ml}$ aliquots of the negative control were placed in four Eppendorf tubes. Cells were pelleted, supernatant was aspirated, and the cell pellets were lysed in $0.25 \mathrm{ml} 0.5 \mathrm{M} \mathrm{NaOH}$. Cell lysates were then frozen at $-20^{\circ} \mathrm{C}$ until all time points were collected. The remaining cultures were incubated at $37^{\circ} \mathrm{C}$. Tissue culture medium was replaced every $48 \mathrm{~h}$, and cells were harvested at the designated time points by aspirating the medium and lysing the monolayers in $0.5 \mathrm{M} \mathrm{NaOH}(0.25 \mathrm{ml} /$ well), and freezing at $-20^{\circ} \mathrm{C}$. The DNA contents of the monolayers were quantified by Hoechst 33258 fluorescence ${ }^{19,29}$ using a Dynex Fluorolite 1000 fluorescence plate reader (Dynex Technologies, Inc., Chantilly, VA, USA). DNA concentrations were calculated as the mean \pm S.D. for triplicate determinations.

\section{${ }^{51} \mathrm{Cr}$-release assay for cytotoxicity}

The original assay ${ }^{30}$ was modified as follows. PC cell lines were removed from culture flasks by incubating at $37^{\circ} \mathrm{C}$ in a trypsin-free chelating solution (135 mM NaCl, $5 \mathrm{mM} \mathrm{KCl}, 20 \mathrm{mM}$ HEPES, and $1.5 \mathrm{mM}$ EDTA, $\mathrm{pH}$ 7.4). For each target cell line, $10^{6}$ cells were pelleted and resuspended in $1 \mathrm{ml} \mathrm{RPMI} 1640$ supplemented with $7.5 \%$ FBS and $10 \mathrm{mM}$ HEPES buffer. Cells were labeled with $100 \mu \mathrm{Ci}{ }^{51} \mathrm{Cr}$ as sodium chromate (ICN Pharmaceuticals Inc., Irvine, CA, USA) in this medium for $1 \mathrm{~h}$ at $37^{\circ} \mathrm{C}$. Unincorporated ${ }^{51} \mathrm{Cr}$ was removed by washing twice in $10 \mathrm{ml}$ medium, incubating for $1 \mathrm{~h}$ in fresh medium at $37^{\circ} \mathrm{C}$, and washing again. Five thousand radiolabeled target cells in $100 \mu$ l were placed in each well of a round-bottomed 96-well tissue culture plate. Effector cells (K562-hFasL or K562-neo) were added at $10: 1,1: 1$, or $0.1: 1$ ratios to the target cells, also in a volume of $100 \mu \mathrm{l}$ per well. The plates were incubated for $16-20 \mathrm{~h}$ at $37^{\circ} \mathrm{C}$ after which the cells were pelleted by centrifugation and $100 \mu \mathrm{l}$ of cell-free supernatant were transferred to separate tubes for quantification of radioactivity using a gamma counter. Per cent specific lysis was calculated using the following formula: $(e-s / m-s) \times 100$, were $e, s$ and $m$ equal the amount of radioactivity released from $P C$ cells incubated with effector cells (experimental lysis), with $100 \mu \mathrm{l}$ medium instead of effector cells (spontaneous lysis), or with $100 \mu \mathrm{l} 1 \%$ Triton X-100 (maximum lysis), respectively. Results are presented as the mean \pm S.D. for triplicate samples.

\section{Flow cytometric quantification of Fas receptor expression}

Cell monolayers were harvested at $50-60 \%$ confluency using the trypsin-free chelating solution described for ${ }^{51} \mathrm{Cr}$-release experiments.

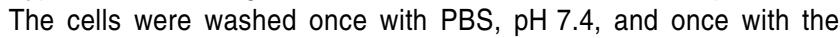
blocking solution (PBS with $5 \%$ goat serum and $0.1 \%$ sodium azide). $5 \times 10^{5}$ cells were placed in $1.5 \mathrm{ml}$ microcentrifuge tubes, pelleted and resuspended in $200 \mu \mathrm{l}$ of blocking solution containing either $2 \mu \mathrm{g} / \mathrm{ml}$ FITC-conjugated mouse IgG1 anti-human Fas receptor (clone DX2, Pharmingen, San Diego, CA, USA), or $2 \mu \mathrm{g} / \mathrm{ml}$ FITC-conjugated isotype control (clone DAK-G01, Dako Laboratories, Carpenteria, CA, USA). Samples were gently mixed and incubated at room temperature for $15 \mathrm{~min}$, protected from light. Cells were washed once with PBS, fixed with $1 \%$ formaldehyde in PBS for $5 \mathrm{~min}$, washed again with PBS and resuspended in $0.5 \mathrm{ml}$ PBS for fluorescence analysis (University of Colorado Cancer Center Flow Cytometry Core, UCHSC).

\section{Prostate tumor growth in athymic nude mice}

ALVA-31 and TSU-Pr1 cells were incubated alone (controls) or with recombinant adenoviruses (10 p.f.u./cell) for $60 \mathrm{~min}$ as described for the growth assays. The cells were washed twice with tissue culture medium and once with PBS. Cell pellets were resuspended in PBS to yield a concentration of $3 \times 10^{6}$ cells per $100 \mu$ l (TSU-Pr1) or $2 \times 10^{6}$ cells per $100 \mu$ l (ALVA-31). For each PC cell line, four male $\mathrm{Nu} / \mathrm{Nu}$ mice (National Cancer Institute, Bethesda, MD, USA), 6-8-weeks-old, were injected intradermally with $100 \mu \mathrm{l}$ of the cell suspensions in a total of five sites per mouse including both shoulders, both hips, and the center of the back. Each mouse, therefore, harbored three control tumors, one transduced with Ad-mFasL, and one transduced with AdEGFP.

When control tumors were approximately $0.5 \mathrm{~cm}$ in size (approximately 10 days later), two control tumors per mouse were injected in the centers with $50 \mu$ l free Ad-mFasL or Ad-EGFP virus $\left(5 \times 10^{8} \mathrm{pfu} / \mathrm{ml}\right)$ to determine if regression or rejection could be initiated in an already established tumor. One mouse was then sacrificed $24 \mathrm{~h}$ later to histologically examine the tumors. Tumor sizes were measured using calipers. Two to six weeks after the start of the experiment, the remaining mice were sacrificed and the excised tumors were fixed in formalin and embedded in paraffin. Tissue sections were then stained with hematoxylin and eosin (Histology Laboratory, Department of Surgical Pathology, UCHSC). Histologic analyses were completed with the assistance of two objective and trained Pathologists, Drs. John Ryder and Rosina DeCampo (Department of Pathology, UCHSC).

\section{Acknowledgements}

This work was funded in part by a grant from the Cancer League of Colorado to T. Hedlund, as well as by the following grants from the USPHS-NIH: NRSA training grant T32-AI07405 to T. Hedlund through the Department of Immunology; HL58344 to J. Schaack; CA-78631 to A. Kraft; Al40607 and Al40394 to R. Duke; and CA-46934 through the Immunology Core Laboratory from the University of Colorado Cancer Center.

\section{References}

1. Itoh N, Yonehara S, Ishii A, Yonehara M, Mizushima S, Sameshima M, Hase A, Seto Y and Nagata S (1991) The polypeptide encoded by the cDNA for humancell surface antigen Fas can mediate apoptosis. Cell 66: 233-243

2. Oehm A, Behrmann I, FalkW, Pawlita M, Maier G, Klas C, Li-Weber M, Richards S, Dhein J, Trauth BC and et al. (1992) Purification and molecular cloning of the APO-1 cell surface antigen, a member of the tumor necrosis factor/nerve growth factor receptor superfamily. Sequence identity with the Fas antigen. J. Biol. Chem. 267: 10709-10715

3. Suda T, Takahashi T, Golstein P and Nagata S (1993) Molecular cloning and expression of the Fas ligand, a novel member of the tumor necrosis factor family. Cell 75: $1169-1178$

4. Nagata S and Golstein P (1995) The Fas death factor. Science 267: 1449-1456

5. Miyawaki T, Uehara T, Nibu R, Tsuji T, Yachie A, Yonehara S and Taniguchi N (1992) Differential expression of apoptosis-related Fas antigen on lymphocyte subpopulations in human peripheral blood. J. Immunol. 149: 3753-3758

6. Owen-Schaub LB, Yonehara S, Crump WLD and Grimm EA (1992) DNA fragmentation and cell death is selectively triggered in activated human lymphocytes by Fas antigen engagement. Cell. Immunol. 140: 197-205

7. Bellgrau D, Gold D, Selawry H, Moore J, Franzusoff A and Duke RC (1995) A role for CD95 ligand in preventing graft rejection. Nature 377: 630-632

8. Xerri L, Devilard D, Hassoun J, Mawas C and Birg F (1997) Fas ligand is not only expressed in immune privileged human organs but is also coexpressed with Fas in various epithelial tissues. Mol. Pathol. 50: 87-91 
9. Leithauser F, Dhein J, Mechtersheimer G, KoretZ K, Bruderlein S, Henne C, Schmidt A, Debatin KM, Krammer PH and Moller P (1993) Constitutive and induced expression of APO-1, a new member of the nerve growth factor/tumor necrosis factor receptor superfamily, in normal and neoplastic cells. Lab. Invest. 69: $415-429$

10. French LE, Hahne M, Viard I, Radlgruber G, Zanone R, Becker K, Muller C and Tschopp J (1996) Fas and Fas ligand in embryos and adult mice: ligand expression in several immune-privileged tissues and coexpression in adult tissues and characterized by apoptotic cell turnover. J. Cell Biol. 133: 335-343

11. Isaacs JT, Lundmo PI, Berges R, Martikainen P, Kyprianou N and English HF (1992) Androgen regulation of programmed death of normal and malignant prostatic cells. J. Androl. 13 457-464

12. Rotello RJ, Lieberman RC, Lepoff RB and Gerschenson LE (1992) Characterization of uterine epithelium apoptotic cell death kinetics and regulation by progesterone and RU 486. Am. J. Pathol. 140: 449-456

13. Suzuki A, Enari M, Eguchi $Y$, Matsuzawa A, Nagata S, Tsujimoto $Y$ and Iguchi $T$ (1996a) Involvement of Fas in regression of vaginal epithelia after ovariectomy and during an estrous cycle. EMBO J. 15: 211-215

14. Suzuki A, Matsuzawa A and Iguchi T (1996b) Down regulation of Bcl-2 is the first step on Fas-mediated apoptosis of male reproductive tract. Oncogene 13: 31 37

15. Rokhlin OW, Bishop GA, Hostager BS, Waldschmidt TJ, Sidorenko SP, Pavloff N, Kiefer MC, Umansky SR, Glover RA and Cohen MB (1997) Fas-mediated apoptosis in human prostatic carcinoma cell lines. Cancer Res. 57: 1758-1768

16. Uslu R, Borsellino N, Frost P, Garban $\mathrm{H}, \mathrm{Ng} \mathrm{C}$, Mizutani $\mathrm{Y}$, Belldegrun $\mathrm{A}$ and Bonavida B (1997) Chemosensitization of human prostate carcinoma cell lines to anti-Fas-mediated apoptosis. Clin. Cancer Res. 3: 963-972

17. Hedlund T, Duke R, Schleicher M and Miller G (1998) Fas-mediated apoptosis in seven human prostate cancer cell lines: correlation with tumor stage. Prostate 36: $92-101$

18. Tewari M and Dixit VM (1995) Fas- and tumor necrosis factor-induced apoptosis is inhibited by the poxvirus crmA gene product. J. Biol. Chem. 270: 3255-3260

19. Hedlund TE and Miller GJ (1994) A serum-free defined medium capable of supporting growth of four established human prostatic carcinoma cell lines. Prostate 24: 221-228

20. Lozzio C and Lozzio B (1975) Human chronic myelogenous leukemia cell line with positive Philadelphia chromosome. Blood 45: 321 - 334

21. Graham FL, Smiley J, Russell WC and Nairn R (1977) Characteristics of a human cell line transformed by DNA from human adenovirus type 5 . J. Gen. Virol. 36 $59-74$

22. Duke RC, Newell E, Schleicher M, Meech S and Bellgrau D (1998) Transplantation of cells and tissues expressing Fas ligand. Transplantation Proc. (in press)

23. Jordan M, Schallhorn A and Wurm FM (1996) Transfecting mammalian cells: optimization of critical parameters affecting calcium-phosphate precipitate formation. Nucl. Acids Res. 24: 596-601

24. Rouvier E, Luciani MF and Golstein P (1993) Fas involvement in $\mathrm{Ca}(2+)$ independent T cell-mediated cytotoxicity. J. Exp. Med. 177: 195-200

25. Zhang G, Gurtu V and Kain SR (1996) An enhanced green fluorescent protein allows sensitive detection of gene transfer in mammalian cells. Biochem. Biophys. Res. Commun. 227: 707-711

26. Gomez-Foix AM, Coats WS, Baque S, Alam T, Gerard RD and Newgard CB (1992) Adenovirus-mediated transfer of the muscle glycogen phosphorylase gene into hepatocytes confers altered regulation of glycogen metabolism. J. Biol. Chem. 267: 25129-25134

27. Schaack J, Langer S and Guo X (1995) Efficient selection of recombinant adenoviruses by vectors that express beta-galactosidase. J. Virol. 69: 39203923
28. Hardy S, Kitamura M, Harris-Stansil T, Dai Y and Phipps ML (1997) Construction of adenovirus vectors through Cre-lox recombination. J. Virol. 71: 1842-1849

29. Labarca C and Paigen K (1980) A simple, rapid, and sensitive DNA assay procedure. Analytical Biochemistry 102: 344-352

30. Duke R, Chervenak R and Cohen J (1983) Endogenous endonuclease-induced DNA fragmentation: An early event in cell-mediated cytolysis. Proc. Natl. Acad. Sci. USA 80: 6361-6365

31. McClinton S, Miller ID and Eremin O (1990) An immunohistochemical characterisation of the inflammatory cell infiltrate in benign and malignant prostatic disease. Brit. J. Cancer 61: 400-403

32. Wickham TJ, Mathias P, Cheresh DA and Nemerow GR (1993) Integrins alphav v beta 3 and alpah $v$ beta 5 promote adenovirus internalization but not virus attachment. Cell 73: 309-319

33. Huang S, Kamata T, Takada Y, RuggeriZMand Nemerow GW (1996) Adenovirus interaction with distinct integrins mediates separate events in cell entry and gene delivery to hematopoietic cells. J. Virol. 70: 4502-4508

34. Witkowski CM, Rabinovitz I, Nagle RB, Affinito KS and Cress AE (1993) Characterization of integrin subunits, cellular adhesion and tumorgenicity of four human prostate cell lines. J. Cancer Res. Clin. Oncol. 119: 637-644

35. Haywood-Reid PL, Zipf DR and Springer WR (1997) Quantification of integrin subunits on human prostatic cell lines - comparison of nontumorigenic and tumorigenic lines. Prostate 31:1-8

36. Muruve DA, Nicolson AG, Manfro RC, Strom TB, Sukhatme VP and Libermann TA (1997) Adenovirus-mediated expression of Fas ligand induces hepatic apoptosis after Systemic administration and apoptosis of ex vivo-infected pancreatic islet allografts and isografts. Hum. Gene Ther, 8: 955-963

37. Pajouh MS, Nagle RB, Breathnach R, Finch JD, Brawer MK and Bowden GT (1991) Expression of metalloproteinase genes in human prostate cancer. J. Cancer Res. Clin. Oncol. 117: 144-150

38. Roth BJ (1996) New therapeutic agents for hormone refractory prostate cancer. Sem. Oncol. 23: 49-55

39. Fife RS, Sledge GW and Proctor C (1997) Effects of vitamin D3 on proliferation of cancer cells in vitro. Cancer Lett. 120: 37-41

40. Suda T, Hashimoto H, Tanaka M, Ochi T and Nagata S (1997) Membrane Fas ligand kills human peripheral blood T lymphocytes and soluble Fas ligand blocks the killing. J. Exp. Med. 186: 2045-2050

41. Tanaka M, Itai T, Adachi M and Nagata S (1998) Downregulation of Fas ligand by shedding. Nature Med. 4: 31-36

42. Schneider P, Holler N, Bodmer JL, Hahne M, Frei K, Fontana A and Tschopp J (1998) Conversion of membrane-bound Fas (CD95) ligand to its soluble form is associated with downregulation of its proapoptotic activity and loss of liver toxicity. J. Exp. Med. 187: 1205-1213

43. Peitsch MC and Tschopp J (1995) Comparative molecular modelling of the Fasligand and other members of the TNF family. Mol. Immunol. 32: 761-772

44. Liu WY, Rubin MA, Omene C, Lederman S and Stein CA (1998) Fas ligand is constitutively secreted by prostate cancer cells in vitro. Clin. Cancer Res. 4: $1803-1811$

45. Arai H, Gordon D, Nabel E and Nabel G (1997) Gene transfer of Fas ligand induces tumor regression in vivo. Proc. Natl. Acad. Sci. USA 94: 13862-13867

46. Schmid HP, McNeal JE and Stamey TA (1993) Observations on the doubling time of prostate cancer. The use of serial prostate-specific antigen in patients with untreated disease as a measure of increasing cancervolume. Cancer 71:2031 2040 